\title{
ATRIBUTOS FÍSICOS E CARBONO ORGÂNICO EM SOLO SOB CERRADO CONVERTIDO PARA PASTAGEM E SISTEMA MISTO
}

\section{Physical attributes and organic carbon in soil under brazilian Savannah converted to pasture and mixed system}

Thalita Mendes Resende Universidade Federal de Uberlândia, Uberlândia, Minas Gerais, Brasil thalitamresende@yahoo.com.br

Vania Rosolen Universidade Estadual Paulista, Rio Claro, São Paulo, Brasil vrosolen@rc.unesp.br

Martial Bernoux Institut de Recherche pour le Développement, Paris, França martial.bernoux@ird.fr

Jorge Luís Silva Brito Universidade Federal de Uberlândia, Uberlândia, Minas Gerais, Brasil jbrito@ufu.br

Elias Nascentes Borges Universidade Federal de Uberlândia, Uberlândia, Minas Gerais, Brasil elias@ufu.br

Fabrício Pelizer Almeida Universidade Federal de Uberlândia, Uberlândia, Minas Gerais, Brasil fabricio.pelizer@gmail.com

RESUMO: A pastagem é uma importante atividade econômica no Cerrado. A partir das décadas de 1970 e 1980 , programas para melhoria das pastagens introduziu gramíneas principalmente do gênero Brachiaria. Quando manejada adequadamente, a pastagem é reconhecida como eficiente em sequestrar carbono e contribuir com a mitigação das concentrações de gases do efeito estufa na atmosfera, condição que será atingida em função do tempo de uso e das propriedades de cada tipo de solo. A microrregião de Uberlândia no Triângulo Mineiro (MG) é tradicionalmente ligada à pastagem. No entanto, as condições geológicas, naturais do solo e do clima são fatores que podem acelerar a degradação dos solos quando a pastagem não é manejada adequadamente. $\mathrm{O}$ objetivo deste artigo foi comparar as mudanças das propriedades 
do solo e a incorporação de C em áreas recobertas com [1] Cerrado arbóreo natural, [2] sistema misto convertido inicialmente ao uso agrícola e os últimos 15 anos de pastagem, e [3] pastagem cultivada há 30 anos. Os resultados mostram a influência de uso sobre a dinâmica da matéria orgânica, e avalia o potencial de degradação dos solos mediante a atividade, o manejo e o tempo de uso do solo.

Palavras-Chaves: Densidade; matéria orgânica; sistemas agropecuários; Cerrado.

ABSTRACT: The pasture is an important economic activity in the brazilian Savannah. From the 1970s and 1980s, the implementation of the programs to improve the productivity of pastures, was introduced grasses, especially Brachiaria. When handled properly, the pasture is an efficient method to contribute to carbon sequestration and to the mitigation of concentrations of greenhouse gases in the atmosphere, which are dependent to the time of the land-use and the properties of each soil type. The microregion of Uberlandia in Triangulo Mineiro (MG) is traditionally linked to the pasture. However, the geological and natural soil conditions and climate are factors that can accelerate soil degradation when pasture is not handled properly. The objective of this article was to compare the changes of soil properties and the incorporation of $\mathrm{C}$ in covered areas [1] natural brazilian Savannah, [2] mixed system initially converted to agricultural use and the last 15 years of pasture, and [3] pasture cultivated with 30 years of implantation. The results show the influence of the land use on organic matter dynamics, and evaluate the potential degradation of soil by the activity, management and time of land use.

Keywords: Bulk density; organic matter; agricultural systems; brazilian Savannah.

\section{INTRODUÇÃO}

Historicamente, a pastagem sempre foi uma atividade econômica importante no Cerrado. Inicialmente, de forma extensiva, era desenvolvida após o corte e a queima da vegetação nativa. Nas décadas de 1970 e 1980, programas para melhoria das pastagens introduziu gramíneas exóticas e de pequena exigência nutricional, principalmente espécies do gênero Brachiaria, que potencializou o aumento da biomassa, a qualidade nutricional para os rebanhos e a conservação dos solos frágeis do Cerrado (MARTHA JÚNIOR; VILELA, 2002; BAYER et al., 2006; MAIA et al., 2009). Por ser reconhecido mundialmente como área de expansão da fronteira agrícola, o Cerrado é um mosaico que conjuga agricultura intensiva e pastagem, entremeados com fragmentos da vegetação nativa, sensivelmente ameaçados pelo rápido avanço da conversão, uma vez que apenas $2,85 \%$ da área total são unidades de conservação de proteção integral (MMA, 2014).

As pastagens cultivadas ocupam aproximadamente 150 milhões de hectares no Brasil dos quais o cerrado contribui com cerca de 56 milhões de hectares, ou seja, 37\% do total (BUSTAMANTE et al., 2012), incrementadas pela introdução de cultivares de plantas forrageiras selecionadas e/ou melhoradas que resultaram em ganhos expressivos na taxa de lotação, no desempenho e na produtividade animal (BOIN, 1998). O manejo inadequado do sistema solo - planta e forrageira - animal, em pastejo, geralmente é o que pode determinar a degradação da pastagem (MARTHA JÚNIOR; CORSI, 2001). A redução da fertilidade do solo para índices inferiores aos encontrados sob vegetação nativa (LILIENFEIN et al., 2003) e o rápido declínio dos serviços ambientais do bioma são comumente observados (BRANNSTROM et al., 2008).

A compreensão e a quantificação do impacto do uso e manejo do solo na sua qualidade física são fundamentais no desenvolvimento de sistemas agrícolas sustentáveis (DEXTER; YOUNGS, 1992). A degradação física destes solos está relacionada com a redução nos teores de matéria orgânica (CARDOSO et al., 1992), resultando em menor retenção de água e estabilidade dos agregados do solo (COSTA; COELHO, 1990; COSTA et al., 1997). No Brasil, têm sido feitos alguns estudos avaliando as mudanças nas propriedades dos solos utilizando o solo sob mata como referência (SILVA; RIBEIRO, 1992; ARAÚJO et al., 1998; SANCHES et al., 1999; BORGES et al., 1999). A introdução 
de sistemas agrícolas em substituição às florestas causa um desequilíbrio no ecossistema, modificando as propriedades do solo (COSTA et al., 2008), cuja intensidade varia com as condições de clima, uso e manejos adotados e a natureza do solo. Com o uso intensivo dos solos, geralmente ocorre a deterioração das suas propriedades físicas (COOTE; RAMSEY, 1983). Modificações na densidade e na porosidade do solo podem variar consideravelmente, dependendo da textura, dos teores de matéria orgânica do solo e da freqüência de cultivo (HAJABBASI et al., 1997).

A pastagem degradada é reconhecidamente fonte de gases do efeito estufa e a mitigação dos efeitos por meio de sua recuperação exige elevados investimentos em adubação e fertilizantes, o que pode tornar a prática financeiramente impeditiva, além do que alguns insumos podem ser agentes de liberação de gases (CONANT et al., 2001; BERNOUX et al., 2003). Pastagem quando bem manejadas estocam $\mathrm{C}$ enquanto que as degradadas liberam $\mathrm{C}$ na forma de $\mathrm{CO}_{2}$ e amplia o estado de degradação dos solos (CORAZZA et al., 1999; JANTALIA et al., 2007; BAYER et al., 2010). Associados ao manejo, outros fatores ambientais e antrópicos interferem na complexidade do aumento ou redução do estoque de C do solo após a conversão da vegetação nativa do cerrado em cultivos comerciais, como por exemplo, o tipo de solo, o tipo de rotação das culturas, as condições climáticas e a intensidade do cultivo e prática adotada (CARVALHO et al., 2014). De acordo com Machado (2005), os processos responsáveis pelas perdas de carbono no solo são a erosão, a decomposição, a volatilização e a lixiviação. Ao mesmo tempo, os três principais processos responsáveis pelo seqüestro de carbono nos solos são a humificação, a agregação e a sedimentação. Solos arenosos são particularmente sensíveis à perda de carbono após desmatamento e uso antrópico. A implantação de sistemas de uso que recobrem totalmente o solo, como é o caso da pastagem bem manejada, são eficientes em estabilizar o estoque de $\mathrm{C}$ em patamares satisfatórios após a conversão, apresentando um ganho superior em relação ao estoque inicial, condição que varia em função do tempo de cultivo (MORAES, 1991).

O Triângulo Mineiro (MG) é uma região de produção agrícola que faz uso de tecnologias de produção modernas que reflete o conjunto de políticas governamentais e do setor privado que transformou o Cerrado e inseriu o Brasil no mercado globalizado de commodities a partir da década de 1970 (JEPSON, 2006). A mais recente base de dados disponível (Censo Agropecuário, IBGE, 2006) indica que, apesar do aumento expressivo de cultivos temporários, a pastagem continua a ser uma atividade econômica importante em extensão de área e rendimento dentro do espaço rural do Triângulo Mineiro. A microrregião de Uberlândia é tradicionalmente ligada à pastagem. No entanto, as condições geológicas do solo e as condições climáticas se constituem em fatores determinantes que podem acelerar a degradação dos solos, especialmente a erosão em sulcos, quando a pastagem não é manejada adequadamente.

O objetivo deste trabalho foi comparar as mudanças dos atributos do solo e a incorporação de $\mathrm{C}$ em áreas recobertas com Cerrado arbóreo natural e pastagem cultivada com 30 anos de implantação incluindo, ainda, um sistema misto e complexo representativo na região, com uma área convertida inicialmente ao uso agrícola e nos últimos 15 anos pastagem.

\section{MATERIAL E MÉTODOS}

\section{A área de estudo}

Esta pesquisa foi realizada na Fazenda Cachoeira (1904'06" - 1909'22" S e 48 33'37' 48³6'31" O), localizada no interflúvio dos ribeirões Panga e Douradinho, com acesso no km 37 da BR 497. Geograficamente encontra-se a Sudoeste do município de Uberlândia, Minas Gerais, Brasil (Figura 01). 
Figura 01- Localização da Fazenda Cachoeira no município de Uberlândia, Estado de Minas Gerais, Brasil

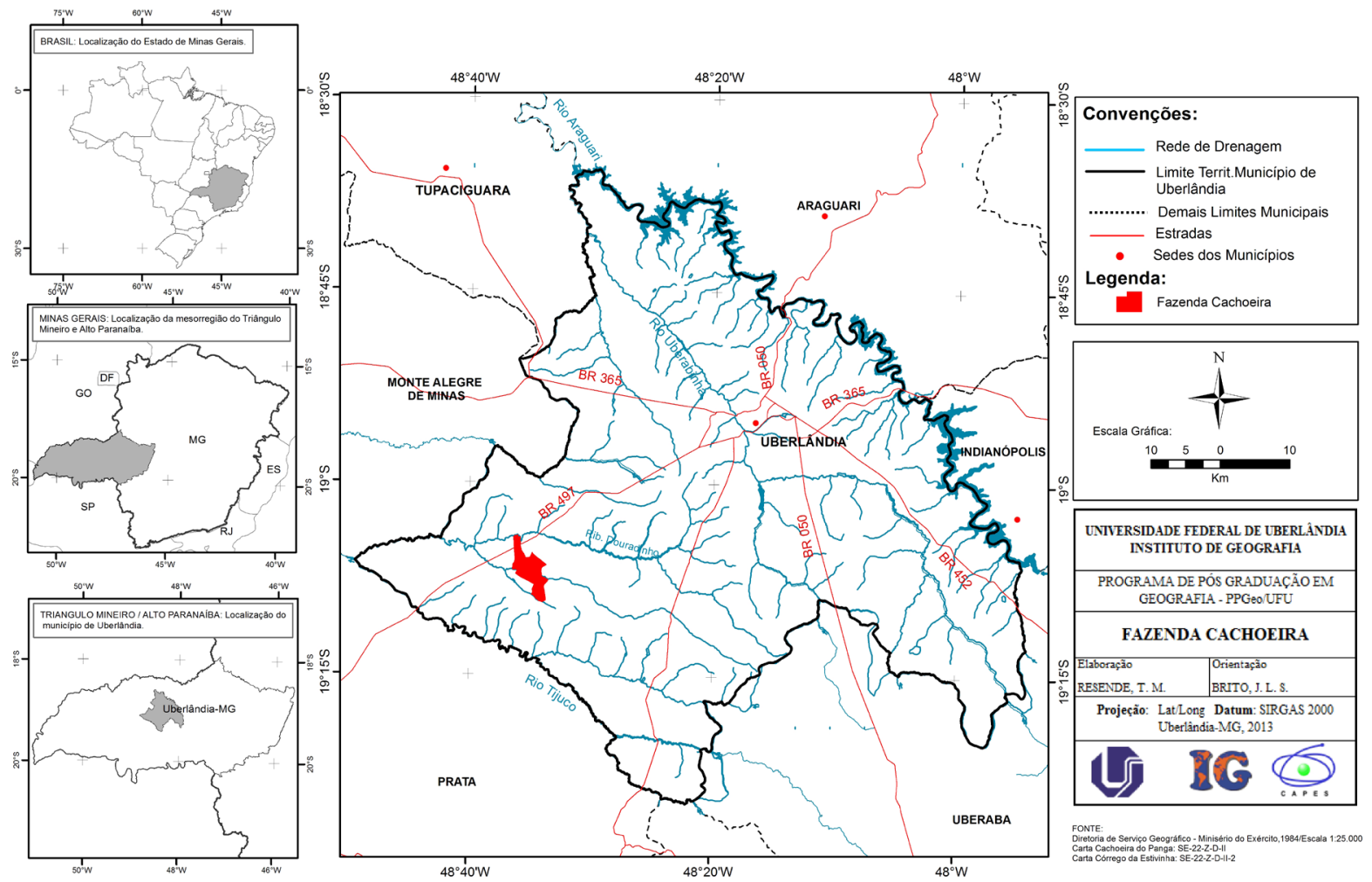

Org. dos autores

\section{Amostragem dos solos}

As amostras foram coletadas em trincheiras, em três profundidades: na superfície entre 0 e 15 $\mathrm{cm}$ e em subsuperfície entre 15 e $30 \mathrm{~cm}$ e entre 30 e $60 \mathrm{~cm}$. A profundidade escolhida está amparada no fato de que os maiores teores de carbono orgânico encontram-se estocados na superfície do solo, onde são esperadas as maiores variações de ganho e perda de matéria orgânica (BERNOUX et al., 2002). Três diferentes usos do solo foram avaliados: [1] solo sob Cerrado nativo (CN), [2] solo sob sistema misto agricultura + pastagem há 15 anos (Agric $+\mathrm{P} 15)$, e [3] solo sob pastagem cultivada há 30 anos (P30). $\mathrm{Na}$ fazenda, são as áreas típicas manejadas pelo proprietário, em uma mesma posição topográfica e contendo os mesmos atributos do solo, com a segurança da espécie de gramínea cultivada e o tipo de manejo adotado.

O cerrado nativo ( sitio de coleta 1 ) representa as propriedades de referência e é caracterizado como cerradão (cerrado arbóreo). Segundo o proprietário, essa área nunca foi desmatada nem queimada e corresponde à Reserva Legal da propriedade (Figura 02). Em 1979, a área P30 (sítio de coleta 3) passou a ser cultivada com pastagem - gramínea Bachiaria ruzizienses (Figura 02). Antes disso, a área era ocupada pela vegetação de Cerrado nativo, manejado com fogo a cada 2 anos. A partir de 1978/79, o solo foi corrigido, arado e gradeado para o cultivo de pastagem.

A área Agric + P15 (sítio de coleta 2) começou a ser desmatada por volta de 1979/80. O corte do Cerrado foi feito gradativamente, ao longo de 10 anos. Durante essa década, observava-se na área um Cerrado Campo Sujo. No início da década de 90, os arbustos restantes foram cortados e o solo foi deixado em 'pousio' durante $2 / 3$ anos e os arbustos removidos mecanicamente sem uso de fogo. Após esse período, foi feita a sistematização do terreno e o solo arado 
e gradeado para o estabelecimento do cultivo de arroz por 2 anos consecutivos, seguido pelo cultivo da soja. A partir do $2^{\circ}$ cultivo da soja a área foi incorporada a pastagem com gramínea Panicum maximum por, aproximadamente, $4 / 5$ anos. A baixa fertilidade do solo e, principalmente a toxicidade por
Al, fez com que a Brachiária invadisse e dizimasse com a Panicum. Assim, nestes 15 anos, a pastagem estabelecida nessa área foram as gramíneas Panicum maximum e Bachiaria ruzizienses por 5 e 10 anos, respectivamente (Figura 02).

Figura 02- Histórico de uso e/ou ocupação das áreas de coleta das amostras de solo na Fazenda Cachoeira município de Uberlândia, Minas Gerais, Brasil (1979, 2000 e 2010)

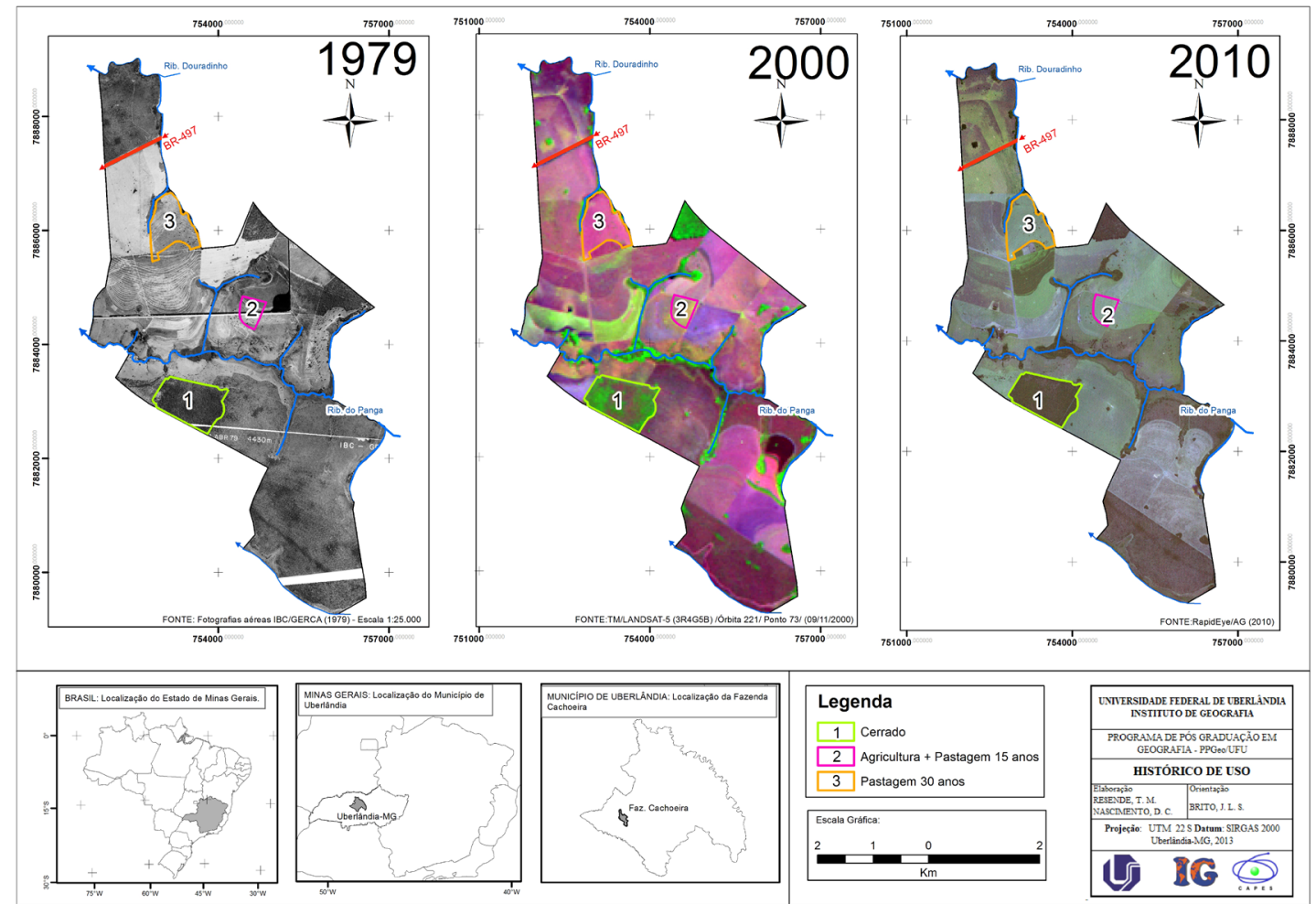

Org. dos autores

As amostras com 4 repetições foram coletadas no mês de abril de 2012 em três profundidades dos perfis de trincheiras abertas aleatoriamente e georreferenciados, totalizando 36 amostras.

\section{Análise Granulométrica}

As amostras foram secas ao ar e peneiradas a $2 \mathrm{~mm}$ para a retirada de $10 \mathrm{~g}$ de TFSA. A dispersão foi feita com uma solução de $50 \mathrm{~mL}$ de $\mathrm{NaOH}$ $0,1 \mathrm{~mol} \mathrm{~L}^{-1}$ e $150 \mathrm{~mL}$ de água deionizada, seguida de agitação mecânica (12.000 rpm) durante $15 \mathrm{~min}$. A fração areia foi obtida por peneiramento e as frações argila e silte por pipetagem, conforme proposto pela Embrapa (1997).

\section{Cálculo da Porosidade Total do solo (Pt)}

Para determinação da porosidade total do solo foram utilizadas as amostras de solo com estrutura indeformada, coletadas com o auxílio do extrator de solo de Uhland (1950) modificado contendo anel volumétrico de metal com volumes e pesos previamente conhecidos. As determinações da macro 
e microporosidade foram feitas em mesa de tensão, como sugerida por Leamer e Shaw (1941), ajustando a coluna de água para $60 \mathrm{~cm}$ de desnível para obter uma tensão de 0,006 Mpa. Antes de levar os anéis com as amostras indeformadas à mesa de tensão, estas foram saturadas em uma bandeja plástica com nível crescente de água até atingir a borda do anel por um período de 12 horas. Após completa saturação foram pesados e colocados sobre a mesa de tensão para a retirada da água contida nos poros maiores do que $0,05 \mathrm{~mm}$, assumidos como macroporos. Após cessar a drenagem por força da tensão equivalente a $60 \mathrm{~cm}$ de coluna de água, os anéis foram então pesados e levados a estufa a $105^{\circ} \mathrm{C}$. Por diferenças entres as sucessivas pesagens, obteve-se o volume de água que dividido pelo volume do anel fornece a quantidade de macro e microporos presente na amostra. A soma destes valores constitui a porosidade total (EMBRAPA, 1997).

\section{Determinação da densidade do solo (Ds)}

A densidade do solo foi obtida por meio do peso seco $(\mathrm{g})$ das amostras indeformadas coletadas em campo, com o auxílio de um anel volumétrico de metal com dimensões e volumes previamente conhecidos (BLAKE; HARTGE, 1986).

\section{Determinação do teor de carbono orgânico total (Ct)}

Para determinação do carbono orgânico total, as amostras de solo foram secas a $50^{\circ} \mathrm{C}$ durante 24 horas. Raiz e outros resíduos vegetais foram removidos. O material vegetal remanescente foi removido por flotação em $\mathrm{HCl} 0,01 \mathrm{M}$ e peneirado a $210 \mu \mathrm{m}$. O Ct foi determinado em um analisador Carlo Erba Analyser CHN-1110, anexado a um espectrômetro de massa Optima Thermo Finnigan, Plus Delta. As análises foram realizadas no Laboratório de Ecologia Isotópica do Centro de Energia Nuclear na Agricultura (CENA/USP). As incertezas analíticas variaram em média $0,3-0,5 \%$. Os teores são expressos em gramas por quilo $\left(\mathrm{g} \cdot \mathrm{Kg}^{-1}\right)$ de solo seco.

\section{Análise estatística}

Os dados coletados foram analisados em delineamento inteiramente casualizado (DIC) em esquema de parcelas subdivididas $3 \times 3 \times 4$ (três sistemas de manejo do solo; três camadas de solo: $0-15 ; 15-30$ e 30-60 cm; quatro repetições). Todos os dados foram submetidos ao teste de F para a análise de variância e o de Tukey para a comparação de médias, todos a $5 \%$, utilizando-se o programa computacional SISVAR versão 5.3 (FERREIRA, 2010).

Somente as interações significativas pelo teste de $\mathrm{F}$, entre os sistemas de manejo das áreas e as camadas de solo, para cada variável analisada ( $\mathrm{Pt}$, Ds e $\mathrm{Ct}$ ), foram demonstradas e discutidas nos resultados. Do mesmo modo, as médias gerais para cada variável, devido ao sistema de manejo das áreas e as camadas de solo, individualmente, também foram submetidas ao teste de Tukey $(\mathrm{P}<0,05)$ e abordadas no trabalho, observando-se a significância pelo teste de F.

\section{RESULTADOS E DISCUSSÃO}

A facilidade em estocar ou perder o carbono do solo pode estar associada à variação no teor e distribuição do tamanho das partículas finas (FELLER; BEARE, 1997; BAI et al., 2012). $\mathrm{Na}$ área de pesquisa, a quantidade de areia, silte e argila variaram de acordo com o uso e/ou ocupação estabelecida, inclusive nas profundidades em que o solo foi coletado (Tabela 01). Embora a textura seja considerada um atributo constante a médio e longo prazos, variações como as ocorridas podem acontecer em função da ação erosiva geológica e antrópica já que a classe textural e a estrutura granular pequena facilitam o processo, mesmo em relevo pouco movimentado.

Os solos sob sistemas agropecuários (Agric $+\mathrm{P} 15$ e P30) possuem constituição relativamente mais arenosa do que o solo sob Cerrado. Em CN, o teor médio de areia na profundidade de 0-15 cm foi de 75,7\%. Em Agric $+\mathrm{P} 15$, o teor médio de areia nessa mesma profundidade foi de $82,5 \%$; e em P30 de 83,4\% (Tabela 01). A conversão do cerrado para um uso antropizado, com mecanização 
para o preparo do solo em sua fase inicial, bem como a prática da calagem e adubação favoreceu a redução na estabilidade dos agregados com movimentação de argila (ADA) tanto verticalmente (Tabela 01) quanto horizontalmente por escoamento superficial. É comum na região do Triângulo Mineiro precipitações superiores a $50 \mathrm{~mm} \mathrm{~h}^{-1}$, valor este muito superior a capacidade de infiltração pelo solo, mesmo para os Latossolos. Desse modo, o solo antropizado com sistemas cultivos resultou em diminuição dos teores de argila na superfície e consequentemente na capacidade de acúmulo de carbono.

Tabela 01- Granulometria em solos sob [1] Cerrado, [2] Agricultura + Pastagem 15 anos, e [3] Pastagem com 30 anos de implantação - Município de Uberlândia (MG), 2012

\begin{tabular}{|l|l|l|l|l|l|}
\hline \multirow{2}{*}{$\begin{array}{l}\text { Identificação } \\
\text { Uso atual }\end{array}$} & \multirow{2}{*}{$\begin{array}{l}\text { Profundidade } \\
(\mathrm{cm})\end{array}$} & \multicolumn{2}{|l|}{$\begin{array}{l}\text { Granulometria } \\
(\text { Média })\end{array}$} & Classificação \\
\cline { 3 - 6 } & & $\begin{array}{l}\text { Areia } \\
\left(\mathrm{g} . \mathrm{Kg}^{-1}\right)\end{array}$ & $\begin{array}{l}\text { Silte } \\
\left(\mathrm{g} . \mathrm{Kg}^{-1}\right)\end{array}$ & $\begin{array}{l}\text { Argila } \\
\left(\mathrm{g} . \mathrm{Kg}^{-1}\right)\end{array}$ & \\
\hline Cerrado & $0-15$ & 757 & 39 & 204 & Franco Argilo Arenoso \\
\hline Cerrado & $15-30$ & 752 & 27 & 221 & Franco Argilo Arenoso \\
\hline Cerrado & $30-60$ & 701 & 56 & 243 & Franco Argilo Arenoso \\
\hline Agric + P15 & $0-15$ & 825 & 50 & 125 & Areia Franca \\
\hline Agric + P15 & $15-30$ & 774 & 32 & 194 & Franco Arenoso \\
\hline Agric + P15 & $30-60$ & 787 & 40 & 173 & Franco Arenoso \\
\hline P30 & $0-15$ & 834 & 54 & 112 & Areia Franca \\
\hline P30 & $15-30$ & 787 & 35 & 178 & Franco arenoso \\
\hline P30 & $30-60$ & 774 & 24 & 202 & Franco arenoso \\
\hline
\end{tabular}

Org. dos autores

A tabela 02 apresenta os valores de porosidade total indicando que somente na área sob Cerrado natural $(\mathrm{CN})$ existe diferença estatisticamente significativa entre as três profundidades analisadas. Para as demais áreas, os valores encontrados não diferem entre si até a profundidade de $60 \mathrm{~cm}$. O comportamento para a mesma variável de estudo $(\mathrm{Pt})$ na profundidade de $0-15 \mathrm{~cm}$, permite afirmar que os valores encontrados em área sob Cerrado natural diferem daqueles encontrados nas demais áreas, apesar de alguma semelhança entre as áreas sob $\mathrm{CN}$ e Agric + P15. Resultados similares, em solo cultivado, foram obtidos por Silva e Ribeiro (1992).

Em relação a Ma, não houve diferença significativa entre os valores encontrados. Já em relação à $\mathrm{Mi}$, houve um decréscimo no volume de microporos nas áreas cultivadas $\left(0,09 \mathrm{e} 0,10 \mathrm{~cm}^{3} \cdot \mathrm{cm}^{-3}\right.$ em Agric $+\mathrm{P} 15$ e P30, respectivamente) em relação aos solos sob $\mathrm{CN}\left(0,14 \mathrm{~cm}^{3} \cdot \mathrm{cm}^{-3}\right)$ sugerindo migração vertical de argila uma vez que este parâmetro é fortemente influenciada pela textura e pelo teor de carbono orgânico e muito pouco influenciada pelo aumento da densidade do solo (ARAÚJO et al., 2004; REINERT; REICHERT, 2006). 
Tabela 02- Porosidade total (Pt), Macroporosidade (Ma) e Microporosidade (Mi) $\left(\mathrm{cm}^{3} \mathrm{~cm}^{-3}\right)$ de um Latossolo sob [1] Cerrado, [2] Agricultura + Pastagem 15 anos, e [3] Pastagem com 30 anos de implantação Município de Uberlândia (MG), 2012(1)

\begin{tabular}{|c|c|c|c|c|c|c|c|c|c|c|c|c|}
\hline \multirow{3}{*}{ Áreas $^{(2)}$} & \multicolumn{4}{|c|}{$\operatorname{Pt}\left(\mathrm{cm}^{3} \mathrm{~cm}^{-3}\right)$} & \multicolumn{4}{|c|}{$\mathrm{Ma}\left(\mathrm{cm}^{3} \mathrm{~cm}^{-3}\right)$} & \multicolumn{4}{|c|}{$\operatorname{Mi}\left(\mathrm{cm}^{3} \mathrm{~cm}^{-3}\right)$} \\
\hline & \multicolumn{12}{|c|}{ Profundidades $^{(3)}$} \\
\hline & $0-15$ & $15-30$ & $30-60$ & $\begin{array}{l}\text { Média } \\
\text { Áreas }\end{array}$ & $0-15$ & $15-30$ & $30-60$ & $\begin{array}{l}\text { Média } \\
\text { Áreas }\end{array}$ & $0-15$ & $15-30$ & $30-60$ & $\begin{array}{l}\text { Média } \\
\text { Áreas }\end{array}$ \\
\hline $\mathrm{CN}$ & $0,42 \mathrm{aA}$ & $\begin{array}{l}0,37 \\
\mathrm{aB}\end{array}$ & $0,37 \mathrm{aB}$ & 0,42 a & $\begin{array}{l}0,27 \\
\mathrm{aA}\end{array}$ & $\begin{array}{l}0,23 \\
\mathrm{aA}\end{array}$ & $\begin{array}{l}0,25 \\
\mathrm{aA}\end{array}$ & 0,25 a & $\begin{array}{l}0,15 \\
\mathrm{aA}\end{array}$ & $\begin{array}{l}0,14 \\
\mathrm{aA}\end{array}$ & $\begin{array}{l}0,12 \\
\mathrm{aA}\end{array}$ & $0,14 a$ \\
\hline $\begin{array}{l}\text { Agric }+ \\
\text { P15 }\end{array}$ & $\begin{array}{l}0,38 \\
a b A\end{array}$ & $\begin{array}{l}0,34 \\
\mathrm{aA}\end{array}$ & $0,36 \mathrm{aA}$ & 0,38 a & $\begin{array}{l}0,29 \\
\mathrm{aA}\end{array}$ & $\begin{array}{l}0,25 \\
\mathrm{aA}\end{array}$ & $\begin{array}{l}0,28 \\
\mathrm{aA}\end{array}$ & 0,27 a & $\begin{array}{l}0,10 \\
\mathrm{aA}\end{array}$ & $\begin{array}{l}0,09 \\
\mathrm{aA}\end{array}$ & $\begin{array}{l}0,08 \\
\mathrm{aA}\end{array}$ & $0,09 b$ \\
\hline P30 & $0,37 \mathrm{bA}$ & $\begin{array}{l}0,35 \\
\mathrm{aA}\end{array}$ & $0,37 \mathrm{aA}$ & 0,37 a & $\begin{array}{l}0,27 \\
\mathrm{aA}\end{array}$ & $\begin{array}{l}0,26 \\
\mathrm{aA}\end{array}$ & $\begin{array}{l}0,26 \\
\mathrm{aA}\end{array}$ & 0,26 a & $\begin{array}{l}0,10 \\
\mathrm{aA}\end{array}$ & $\begin{array}{l}0,09 \\
\mathrm{aA}\end{array}$ & $\begin{array}{l}0,11 \\
\mathrm{aA}\end{array}$ & $0,10 b$ \\
\hline $\begin{array}{l}\text { Média } \\
\text { Prof. }\end{array}$ & $0,39 \mathrm{~A}$ & $\begin{array}{l}0,35 \\
\mathrm{~B}\end{array}$ & $\begin{array}{l}0,37 \\
\mathrm{AB}\end{array}$ & & $0,28 \mathrm{~A}$ & $0,25 \mathrm{~A}$ & $0,26 \mathrm{~A}$ & & $0,12 \mathrm{~A}$ & $0,11 \mathrm{~A}$ & $0,10 \mathrm{~A}$ & \\
\hline $\mathrm{CV}(\%)$ & & $\begin{array}{l}7,5 \\
(\mathrm{~A})\end{array}$ & & $6,1(a)$ & & $\begin{array}{l}12,6 \\
(\mathrm{~A})\end{array}$ & & $\begin{array}{l}13,4 \\
\text { (a) }\end{array}$ & & $\begin{array}{l}32,9 \\
(\mathrm{~A})\end{array}$ & & $\begin{array}{l}19,8 \\
\text { (a) }\end{array}$ \\
\hline
\end{tabular}

${ }^{(1)}$ Médias seguidas da mesma letra minúscula na vertical e maiúscula na horizontal, não diferem entre si pelo teste de Tukey (probabilidade $<0,05$ ); ${ }^{(2)}$ Áreas, onde, $\mathbf{C N}=$ Cerrado natural; Agricultura+P15 = Uso agrícola e 15 anos de Pastagem; P30 = Pastagem há 30 anos; ${ }^{(3)}$ Profundidades de coleta das amostras de solos para cada área de interesse. Fonte: Org. dos autores

Valores mais elevados de porosidade total foram encontrados na área de $\mathrm{CN}$, sendo de 0,39 $\mathrm{cm}^{3} \cdot \mathrm{cm}^{-3}$, enquanto que os sistemas agropecuários apresentaram menores valores, $0,361 \mathrm{~cm}^{3} \cdot \mathrm{cm}^{-3} \mathrm{e}$ $0,364 \mathrm{~cm}^{3} \cdot \mathrm{cm}^{-3}$, respectivamente para as áreas de Agric + P15 e P30 (Tabela 02), podendo ser atribuídos ao não revolvimento do solo no $\mathrm{CN}$ e ao incremento nos teores de argilas nos sistemas antropizados aliado à ausência de rotação de culturas com sistema radicular mais profundo. É reconhecido que as práticas de manejo refletem na forma e frequência dos poros em razão do estresse imposto e da mudança da população de microorganismos e são passíveis de serem identificadas em profundidade, no espaço e no tempo, com redução no sequestro de carbono pelos solos (KAY; VANDENBYGAART, 2002).

Porém, de forma geral, em todos os tratamentos, foram encontrados bons valores de porosidade total, ou seja, valores próximos à $40 \%$, visto que um solo ideal deve apresentar $50 \%$ de volume de poros totais que, na capacidade de campo, teria 33,5\% ocupado pela água e 16,5\% ocupado pelo ar (CAMARGO; ALLEONI, 1997).
As áreas estudadas apresentaram variações nos valores de densidade do solo (Ds), indicando que houve efeito do manejo e/ou adubação sobre este atributo. O uso antrópico aumentou a compactação do solo, especialmente na pastagem que apresentou o maior valor de Ds (Tabela 03). Os resultados encontrados estão de acordo com os obtidos por Islam e Weil (2000), que constataram um valor médio da Ds significativamente maior em área cultivada comparada com solo sob floresta natural. Sanches et al. (1999) constataram que, independentemente da posição de amostragem, a Ds foi maior no sistema cultivado do que no solo sob mata nativa.

O uso principal da densidade do solo é como indicador da compactação, sendo medidos pelas alterações da estrutura e porosidade do solo. A densidade do solo tende a aumentar com o aumento da profundidade no perfil, isto se deve, provavelmente, ao menor teor de matéria orgânica, menor agregação, pouca quantidade de raízes e compactação causada pela massa das camadas superiores (REINERT; REICHERT, 2006). 
Tabela 03- Densidade (Ds, em $\mathrm{g} \mathrm{cm}^{-3}$ ) de um Latossolo sob [1] Cerrado, [2] Agricultura + Pastagem 15 anos, e [3] Pastagem com 30 anos de implantação - Município de Uberlândia (MG), 2012 $2^{(1)}$

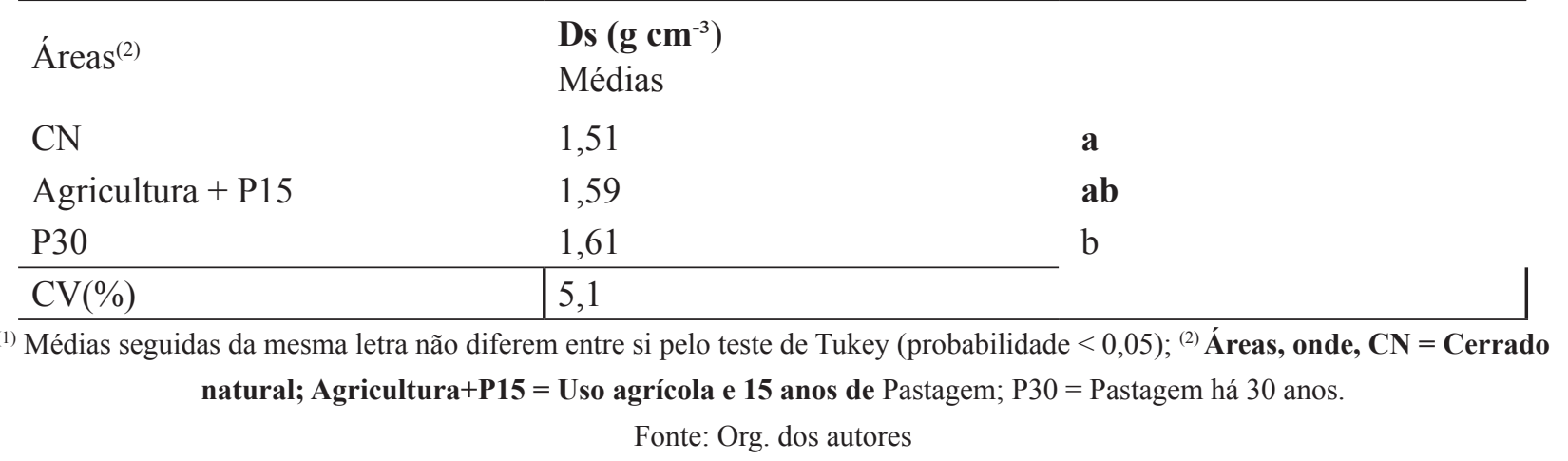

No solo sob pastagem há 30 anos (P30), em função do longo tempo de pisoteamento do gado e do não revolvimento do solo, verificou-se um elevado valor de densidade $(1,61)$, evidenciando que se trata de um solo mais compactado se comparado às demais áreas (1,51 e 1,59 em Agric+ P15 e P30, respectivamente) (Tabela 03). Segundo Leão et al. (2004), o pisoteio animal em toda a superfície e, às vezes, repetidamente no mesmo local promove consideráveis alterações físicas no solo. $\mathrm{Na}$ literatura, são mencionados valores de pressão que variam entre 0,25 e $0,49 \mathrm{MPa}$ para bovinos de 100 a $500 \mathrm{~kg}$, podendo atingir a profundidade de 5 a 10 $\mathrm{cm}$ (PROFFITT et al., 1993). O aumento dos valores de densidade em solos sob sistemas agropecuários é também uma resposta do solo arenoso e pouco estruturado à retirada da vegetação nativa. $\mathrm{O}$ valor mínimo de Ds foi encontrado em solo sob vegetação nativa de Cerrado.
A substituição da vegetação nativa por sistemas cultivados provoca alterações também nos teores de carbono do solo. O valor médio de \% $\mathrm{C}$ determinado entre $0-15 \mathrm{~cm}$ para as diferentes áreas se difere estatisticamente dos valores aferidos nas profundidades de $15-30 \mathrm{~cm}$ e $30-60 \mathrm{~cm}$. Observase que nos primeiros $15 \mathrm{~cm}$, os solos sob vegetação nativa de Cerrado apresentaram teores mais elevados de C em relação aos solos sob sistemas agropecuários. Aumentos no teor de matéria orgânica, notadamente na camada superficial do solo, têm sido associados a sistemas com o mínimo revolvimento do solo (REEVES, 1997 apud TORMENA et al., 2004). À medida que a profundidade aumenta, entre 30 e 60 $\mathrm{cm}$, percebe-se uma aproximação nos valores dos teores de $\mathrm{C}$ tanto em solos sob vegetação nativa de Cerrado, como em solos sob sistemas cultivados (P30 e Agric+P15) (Tabela 04).

Tabela 04- Teor de Carbono (\%C, em g/100g) de um Latossolo sob [1] Cerrado, [2] Agricultura + Pastagem 15 anos, e [3] Pastagem com 30 anos de implantação - Município de Uberlândia (MG), 2012 $2^{(1)}$

\begin{tabular}{|c|c|c|c|c|c|c|c|c|}
\hline \multirow{3}{*}{$\begin{array}{l}\text { Áreas }{ }^{(2)} \\
\mathrm{CN}\end{array}$} & \multicolumn{6}{|c|}{ Profundidades $^{(3)}$} & \multirow{2}{*}{\multicolumn{2}{|c|}{ Médias - Áreas }} \\
\hline & \multirow{2}{*}{$\begin{array}{c}00-15 \\
1,49\end{array}$} & \multicolumn{3}{|c|}{$15-30$} & \multicolumn{2}{|l|}{$30-60$} & & \\
\hline & & $\mathrm{AA}$ & 0,70 & $\mathrm{aB}$ & 0,59 & $\mathrm{aB}$ & 0,93 & $\mathbf{a}$ \\
\hline Agricultura + P15 & 0,86 & $\mathrm{Ca}$ & 0,59 & $\mathrm{aB}$ & 0,52 & $\mathrm{aB}$ & 0,66 & $\mathbf{b}$ \\
\hline P30 & 1,21 & BA & 0,73 & $\mathrm{aB}$ & 0,55 & $\mathrm{aB}$ & 0,83 & $\mathrm{a}$ \\
\hline Médias - Profundidades & 1,19 & $\mathrm{~A}$ & 0,67 & $\mathrm{~B}$ & 0,56 & $\mathrm{~B}$ & & \\
\hline $\mathrm{CV}(\%)$ & 15,89 & & & & & & 15,90 & (a) \\
\hline
\end{tabular}

(1) Médias seguidas da mesma letra minúscula na vertical e maiúscula na horizontal, não diferem entre si pelo teste de Tukey (probabilidade $<0,05)$; ${ }^{(2)}$ Áreas, onde, $\mathbf{C N}=$ Cerrado natural; Agricultura+P15 = Uso agrícola e 15 anos de Pastagem; P30 = Pastagem há 30 anos; ${ }^{(3)}$ Profundidades de coleta das amostras de solos para cada área de interesse. 
O valor médio de \% C aferido nos perfis de solos sob vegetação nativa de Cerrado é estatisticamente semelhante ao valor médio verificado nos perfis de solo sob pastagem cultivada há 30 anos. Os valores encontrados nos perfis de solo sob Agric +P15 difere das demais áreas analisadas. Este comportamento evidencia a estabilidade do teor de carbono ao longo do tempo, visto que os valores obtidos para a pastagem cultivada há 30 anos se assemelha estatisticamente aos \% C encontrados em solos sob vegetação nativa.

Valores mais elevados de \%C em solo sob vegetação de Cerrado podem ser atribuídos ao não revolvimento do solo, permitindo a manutenção do estoque de carbono provenientes da queda de folhas e material vegetal. Em P30 verifica-se valores próximos àqueles aferidos para $\mathrm{CN}$, tanto $\mathrm{em}$ superfície quanto em subsuperfície, o que também pode ser justificado pelo não revolvimento do solo ao longo desses 30 anos de implantação e cultivo da pastagem. O solo sob sistema misto (Agric $+\mathrm{P} 15)$ apresentou os menores valores de $\% \mathrm{C}$ em todas as profundidades, o que pode estar associado a dinâmica de uso e ao revolvimento existente neste solo, tendo em vista que no início da década de 90 foi feita a sistematização (nivelamento) do terreno, a correção, aração e gradagem do solo para o estabelecimento do cultivo de arroz e soja. Após 4 anos de cultivo que foi incorporada a pastagem. A diferente dinâmica de uso dos solos analisados justifica a variação nos valores de $\%$ C (Tabela 04).

A textura do solo também reflete no resultado do $\%$ C. Os solos sob sistemas cultivados (Agric $+\mathrm{P} 15$ e P30) possuem constituição relativamente mais arenosa do que o solo sob Cerrado. Em CN, o teor médio de areia na profundidade de $0-15 \mathrm{~cm}$ foi de 75,7\%, em Agric+P15 de 82,6\%; e em P30 de $83,4 \%$. Os solos arenosos e o não desenvolvimento da macroestrutura são fatores que contribuem para a diminuição da taxa de C. É conhecido que parte da MOS está protegida nos macroagregados, em uma taxa de $10 \%$ em solos cultivados e $19 \%$ em solos não cultivados segundo Beare et al. (1994). Esta capacidade se eleva com o aumento do teor de argila e ausência de cultivo assim como com a freqüência na qual os agregados são susceptíveis de serem destruídos in situ pelo cultivo ou ainda em relação à sua intrínseca estabilidade em água (BALESDENT; CHENU; BALABANE, 2000).

\section{CONCLUSÕES}

As áreas recobertas pela vegetação de Cerrado foram e continuam sendo substituídas principalmente por sistemas agrícolas e pastagens. A conversão da vegetação nativa de Cerrado em sistemas cultivados promove modificações nos atributos físicos, nos teores de matéria orgânica e no estoque de carbono no solo.

Os valores de porosidade total diminuíram e a densidade do solo aumentou mediante a conversão para sistemas agropecuários, quando comparados aos valores determinados para solos sob vegetação nativa.

No solo sob sistema misto (Agric+P15) houve um empobrecimento de teor de carbono $(\mathrm{C} \%)$ especialmente em superfície se comparado à área sob vegetação nativa de Cerrado. No entanto, o solo recoberto com pastagem há 30 anos apresentou valores mais próximos daqueles encontrados na área sob vegetação nativa, tanto em superfície quanto em subsuperfície, o que evidencia a estabilidade do teor de carbono ao longo do tempo.

\section{AGRADECIMENTOS}

ACAPES (Coordenação de Aperfeiçoamento de Pessoal de Nível Superior) pela concessão da bolsa de doutorado-sanduíche (processo $\mathrm{n}^{\circ}$ 1501/2014-03), ao IRD (Institut de Recherche pour le Développement) e ao laboratório UMR Eco\&Sols pela oportunidade da realização do estágio. Ao Sr. Christian R. Hacker por autorizar a pesquisa em sua propriedade e fornecer todas as informações sobre a rotina de uso e manejo do solo.

\section{REFERÊNCIAS}

ARAUJO, Q.R.; COSTA, L.M.; JUCKSCH, I.; FONTES, L.E.F.; REGAZZI, A.J. Alterações nas propriedades físicas de um podzólico vermelhoamarelo da região cacaueira da Bahia, sob diferentes 
coberturas vegetais. Agrotrópica, Itabuna, v.10, p.6978, 1998.

Araújo, M. A.; Tormena, C.A.; Silva, A. P. Propriedades físicas de um latossolo vermelho distrófico cultivado e sob mata nativa. Revista Brasileira de Ciência do Solo, Viçosa, v.28, p.337-345, 2004. DOI: http://dx.doi. org/10.1590/S0100-06832004000200012

BAI, E.; BOUTTON, T.W.; LIU, F.; WU, B.X.; HALLMARK, C.T.; ARCHER, S.R. Spatial variation of $\delta^{13} \mathrm{C}$ and its relation to carbon input and soil texture in a subtropical lowland woodland. Soil Biology \& Biochemistry, v.44, p.102-112, 2012. DOI:10.1016/J. SOILBIO.2011.09.013

BALESDENT, J.; CHENU, C.; BALABANE, B. Relationship of soil organic matter dynamics to physical protection and tillage. Soil \& Tillage Research, Amsterdam, v.53, p.215-230, 2000. DOI:10.1016/S0167-1987(99)00107-5

BAYER, C.; MARTIN-NETO, L.; MIELNICZUK, J.; PAVINATO, A.; DIECKOW, J. Carbon sequestration in two Brazilian Cerrado soils under no-till. Soil \& Tillage Research, Amsterdam, v.86, p.237-245, 2006. DOI:10.1016/J.STILL.2005.02.023

BAYER. L.B.; BATJES, N.H.; BINDRABAN, P.S. Changes in organic carbon stocks upon land use conversion in the Brazilian Cerrado: A review. Agriculture, Ecosystems and Environment, Amsterdam, v.137, p.47-58, 2010. DOI:10.1016/J. AGEE.2010.02.003

BEARE, M. H.; CABRERA, M. L.; HENDRIX, P. F.; COLEMAN, D. C. Aggregate-protected and unprotected organic matter pools in conventionaltillage and no-tillage soils. Soil Science Society American Journal, Madison, v. 58, p. 787-795, 1994. DOI:10.2136/sssaj1994.03615995005800030021x

BERNOUX, M.; CARVALHO, M. C. S.; VOLKOFF, B.; CERRI, C. C. Brazil's soil carbon stocks. Soil Science Society American Journal, Madison, v.66, p.888-896, 2002.

BERNOUX, M.; VOLKOFF, B.; CARVALHO, M.D.C.S.; CERRI, C.C. $\mathrm{CO}_{2}$ emissions fromliming of agricultural soils in Brazil. Global Biogeochemical Cycles, v.17, p.1049, 2003. DOI: 10.1029/2001GB001848

BLAKE, G. R.; HARTGE, K. H. Bulk density. In: Klute, A. (Ed.). Methods of soil analysis. Part 1 Physical and mineralogical methods. Madison: ASA, 1986. p. 363-375.

BOIN, C. Produtividade em gado de corte: evolução e perspectivas. Preços Agrícolas, Piracicaba, v.138, p.8-11, 1998.

BORGES, A.L.; KIEHL, J.C.; SOUZA, L.S. Alteração de propriedades físicas e atividade microbiana de um latossolo amarelo álico após o cultivo com fruteiras perenes e mandioca. Revista Brasileira de Ciência do Solo, Viçosa, v.23, p.1019-1025, 1999.

BRANNSTROM, C.; JEPSON, W.; FILIPPI, A.M.; REDO, D.; XU, Z.; GANESH, S. Land change in the Brazilian Savanna (Cerrado), 1986-2002: comparative analysis and implications for land-use policy. Land Use Policy, Kidlington, v.25, p.579-595, 2008. DOI:10.1016/J.LANDUSEPOL.2007.11.008

BUSTAMANTE, M. M. C.; CARlos, C.A.; SMERALDI, R.; AGUIAR, A. P. D.; BARIONI, L.G.; FERREIRA, L.G.; LONGO, K.; MAY, P.; PINTO, A.S. ; OMETTO, J. P. H. B. Estimating greenhouse gas emissions from cattle raisingin Brazil. Climatic Change, v.115, p.559-597, 2012. DOI: 10.1007/ s10584-012-0443-3

CAMARGO, O. A.; ALLEONI, L. R. F. Compactação do solo e o desenvolvimento de plantas. Piracicaba: ESALQ, 1997. 132p.

CARDOSO, A.; POTTER, R.; DEDECEK, R.A. Estudo comparativo da degradação de solos pelo uso agrícola no Noroeste do estado do Paraná. Pesq. Agropec. Bras., Brasília, v.27, p.349-353, 1992. 
CARVALHO, J.L.N.; RAUCCI, G.S.; FRAZÃO, L.A.; CERRI, C.E.P.; BERNOUX, M.; CERRI, C.C. Croppasture rotation: A strategy to reduce soil greenhouse gas emissions in the Brazilian Cerrado. Agriculture, Ecosystems and Environment, Amsterdam, v.183, p.167-175, 2014. DOI:10.1016/J.AGEE.2013.11.014

Conant, R.T.; Paustian, K.; Elliott, E.T. Grassland management and conversion into grassland: effects on soil carbon. Ecological Applications, v.11, p.343-355, 2001. DOI: $10.3334 / \mathrm{CDIAC} / \mathrm{tcm} .005$

COOTE, D.R.; RAMSEY, J.F. Quantification of the effects of overs 35 years of intensive cultivation on four soils. Can. J. Soil Sci., v.63, p.1-14, 1983.

CORAZZA, E.J.; SILVA, J.E.D.; RESCK, D.V.S.; GOMES, A.C. Comportamento de diferentes sistemas de manejo como fonte ou depósito de carbono em relação à vegetação de Cerrado. Revista Brasileira de Ciência do Solo, Viçosa, v.23, p.425-432, 1999.

COSTA, A.C.S.; COELHO, S.M.R. Efeito do manejo do solo em Latossolo Vermelho-Escuro textura média-LEd2 do Município de Paranavaí-Paraná. II. Estabilidade dos agregados em água. In: CONGRESSO BRASILEIRO E ENCONTRO NACIONAL DE PESQUISA SOBRE CONSERVAÇÃO DE SOLOS, 8., Londrina, 1990. Resumos... Londrina, 1990. p.35.

COSTA, A.C.S.; NANNI, M.R.; JESKE, E. Determinação da umidade na capacidade de campo e ponto de murcha permanente por diferentes metodologias. R. Unimar, Maringá, v.19, p.827-844, 1997.

COSTA, F.S.; BAYER, C.; ZANATTA, J.A.; MIELNICZUK, J. Estoque de carbono orgânico no solo e emissões de dióxido de carbono influenciadas por sistemas de manejo no sul do Brasil. Revista Brasileira de Ciência do Solo, Viçosa, v.32, p.323332, 2008. DOI: http://dx.doi.org/10.1590/S010006832008000100030

DEXTER, A.R.; YOUNGS, I.M. Soil physic toward
2000. Soil \& Tillage Research, Amsterdam, v.24, p.101106, 1992. DOI: 10.1016/0167-1987(92)90095-S

EMPRESA BRASILEIRA DE PESQUISA AGROPECUÁRIA - EMBRAPA. Serviço Nacional de Levantamento e Conservação de Solos. Manual de métodos de análise de solo. 2.ed. Rio de Janeiro, 1997. 212p.

FELLER, C.; BEARE, M.H. Physical control of soil organic matter dynamics in the tropics. Geoderma, Amsterdam, v.7, p.69-116, 1997. DOI:10.1016/ S0016-7061(97)00039-6

FERREIRA, D. F. Programa computacional Sisvar. Versão 5.3. Lavras: UFLA, 2010.

HAJABBASI, M.A.; JALALIAN,A.; KARIMZADEH, H.R. Deforestation effects on soil physical and chemical properties, Lordegan, Iran. Plant Soil, v.190, p.301-308, 1997.

ISLAM, K.R.; WEIL, R.R. Land use effects on soil quality in a tropical forest ecosystem of Bangladesh. Agric. Ecosys. Environ., Amsterdam, v.79, p.9-19, 2000. DOI:10.1016/S0167-8809(99)00145-0

INSTITUTO BRASILEIRO DE GEOGRAFIA E ESTATÍSTICA - IBGE. Censo Agropecuário, 2006. Resultados preliminares. Disponível em: ftp://ftp.ibge. gov.br/Censos/Censo_Agropecuario_2006/. Acesso em: 08 abr. 2014.

JANTALIA, C.P.; RESCK, D.V.S.; ALVES, B.R.J.; ZOTARELLI, L.; URQUIAGA, S.; BODDEY, R.M. Tillage effect on $\mathrm{C}$ stocks of a clayey Oxisol under a soybean-based crop rotation in the Brazilian Cerrado region. Soil \& Tillage Research, Amsterdam, v.95, p.97-109, 2007. DOI:10.1016/J.STILL.2006.11.005

JEPSON, W. Private agricultural colonization on a Brazilian frontier, 1970 e 1980. Journal of Historical Geography, v.32, p.839-863, 2006. DOI:10.1016/J. JHG.2004.12.019

KAY, B.D.; VANDENBYGAART, A.J. Conservation

Soc. \& Nat., Uberlândia, 27 (3): 501-514, set/dez/2015 
tillage and depth stratification of porosity and soil organic matter. Soil \& Tillage Research, Amsterdam, v.66, p.107-118, 2002.

LEAMER, R. W.; SHAW, B. A simple apparatus for measuring non capillary porosity an extensive scale. Journal of American Society of Agronomy, Madison, v.33, p.1003-1008, 1941.

LEÃO, T.P.; SILVA, A.P.; MACEDO, M.C.M.; IMOFF, S.; EUCLIIDES, V.P.B. Intervalo hídrico ótimo na avaliação de sistemas de pastejo contínuo e rotacionado. R. Bras. Ci. Solo, Campinas, v.28, p.415-423, 2004.

LILIENFEIN, J.; WILCKE, W.; VILELA, L.; AYARZA, M.A.; LIMA, S.C. ; ZECK, W. Soil fertility under native Cerrado and pasture in the Brazilian savanna. Soil Science Society of America Journal, Madison, v.67, p.1195-1205, 2003. DOI: 10.2136/ sssaj2003.1195

MACHADO, P. L. O. A. Carbono do Solo e a Mitigação da Mudança Climática Global. Quim. Nova, São Paulo, v. 28, n. 2, p. 329-334, 2005. DOI: http:// dx.doi.org/10.1590/S0100-40422005000200026

MAIA, S.M.F.; OGLE, S.M.; CERRI, C.E.P. ; CERRI, C.C. Effect of grassland management on soil carbon sequestration in Rondônia and Mato Groso states, Brazil. Geoderma, Amsterdam, v.149, p.84-91, 2009. DOI:10.1016/J.GEODERMA.2008.11.023

MARTHA JÚNIOR, G. B.; CORSI, M. Pastagens no Brasil: situação atual e perspectivas. Preços Agrícolas, Piracicaba, v.171, p.3-6, 2001.

MARTHA JÚNIOR, G.B.; VILELA, L. Pastagens no Cerrado: baixa produtividade pelo uso limitado de fertilizantes. Planaltina: Embrapa Cerrados, 2002.

MINISTÉRIO DO MEIO AMBIENTE - MMA. $O$ Bioma Cerrado. Disponível em: http://www.mma.gov. br/biomas/cerrado. Acesso em: 16 abr. 2014.

MORAES, J. F. L. Conteúdos de carbono e tipologia de horizontes nos solos da bacia amazônica. 1991.84 p. Dissertação (Mestrado) - Centro de Energia Nuclear de Agricultura, Universidade de São Paulo, Piracicaba.

PROFFIT, A.P.B.; BENDOTTI, S.; HOWELL, M.R.; EASTHAM, J. The effect of sheep trampling and grazing on soil physical properties and pasture growth for a RedBrown Earth. Austr. J. Agr. Res., v.44, p.317331, 1993. DOI: 10.1071/AR9930317

REINERT, D.J.; REICHERT, J.M. Coluna de areia para medir a retenção de água no solo - protótipos e teste. Ciência Rural, Santa Maria, v.36, p.1931-1935, 2006.

SANCHES, A.C.; SILVA, A.P.; TORMENA, C.A.; RIGOLIN A.T. Impacto do cultivo de citros em propriedades químicas, densidade do solo e atividade microbiana de um Podzólico Vermelho-Amarelo. Revista Brasileira de Ciência do Solo, Viçosa, v.23, p.91-99, 1999.

SILVA, M.S.L.; RIBEIRO, M.R. Influência do cultivo contínuo da cana-de-açúcar em propriedades morfológicas e físicas de solos argilosos de tabuleiro no estado de Alagoas. Revista Brasileira de Ciência do Solo, Campinas, v.16, p.397-402, 1992.

TORMENA, C.A.; FRIEDRICH, R.; PINTRO, J.C.; COSTA, A.C.S.; FIDALSKI, J. Propriedades físicas e taxa de estratificação de carbono orgânico num Latossolo Vermelho após dez anos sob dois sistemas de manejo. Revista Brasileira de Ciência do Solo, Viçosa, v.28, p.1023-1031, 2004. DOI: http://dx.doi. org/10.1590/S0100-06832004000600011.

UHLAND, R. E. Physical properties of soils as modified by crops and management. Soil Science Society of American Journal, Madison, v.14, p.361366, 1950. 